\title{
Autonomous Coordination Technology through Community Organization for Resource Utilization
}

\author{
Titichaya THANAMITSOMBOON $^{\dagger \text { a) }}$, Kotaro HAMA ${ }^{\dagger}$, Riyako SAKAMOTO ${ }^{\dagger}$, Nonmembers, , \\ Xiaodong LU $\mathbf{U}^{\dagger}$, Member, and Kinji MORI ${ }^{\dagger}$, Fellow
}

\begin{abstract}
SUMMARY The challenge in resource utilization under dynamic environment is how to utilize appropriate resources to the right users at the right time and the right location. In conventional system, centralized management system is applied but it tends to congest when user requests increase or resources rapidly move. Therefore, this paper proposes Autonomous Coordination Technology (ACT) through community organization for resource utilization. In ACT, a node which has surplus resources autonomously constructs community with a surplus-level based size and distributes resources to members which are deficient in resources. ACT consists of autonomous coordination within community and among communities. According to community organization, online property and flexibility can be satisfied. However, it is difficult to achieve service provision timeliness and resource allocation operatability in the mean time. Thus, ACT includes successive transportation method, and autonomous resource allocation which dynamic decision is made by a tradeoff between timeliness and operatability. As a result, the service assurance in terms of timeliness and operatability can be assured. The effectiveness of proposed technology is affirmed through the simulation of taxi dispatching application in terms of response time and standard deviation versus user rates.

key words: autonomous decentralized community system, resource utilization, autonomous coordination
\end{abstract}

\section{Introduction}

In innovative world, the knowledge of wireless communication infrastructures have been rapidly developed [1], [2]. Nonetheless, creating new technologies is not the only challenge. For service provider, searching for the precise service and allocate appropriate resources to satisfy the right users at right time and right place is a significant mission.

Referred to public institute, MLIT [3], Intelligent Transport System (ITS) has been promoted. In transportation system [4]-[6], promptly providing appropriate resources to users and effective utilizing resources are significant. One direction to manage this system is the conventional system which based on centralized management [5], [6]. Yet, there is a limitation in conventional system; it is that management center must know the whole information in the system then it can allocate the most appropriate resource to provide to user. However, if management center fails, the whole system will also be corrupted. Because of its own traits, centralized system is effective in a smallscale system but tends to congest in a large-scale system.

Manuscript received April 25, 2010.

Manuscript revised August 26, 2010.

${ }^{\dagger}$ The authors are with the Department of Computer Science, Tokyo Institute of Technology, Tokyo, 152-8552 Japan.

a)E-mail: titichaya@mori.cs.titech.ac.jp

DOI: 10.1587/transinf.E94.D.11
Moreover, its efficiency also deteriorates due to dynamic situations, i.e. unpredictable user requests, random resource mobility, etc. Hence, an approach on decentralized system aimed to achieve system requirements becomes a promising alternative way.

Based on the concept of Autonomous Decentralized System (ADS) [7], Autonomous Decentralized Community System (ADCS) has been proposed [8]-[13]. Community is autonomously formed with local majority information sharing among same-preference members and each member coordinates to one another autonomously. However, resource utilization system differs from information service system. Community constructs not only for information sharing but also for physical resource utilization. Therefore, this paper proposes Autonomous Coordination Technology (ACT) through community organization for resource utilization. A node which has surplus resources autonomously constructs a community (defined as a group of base stations and community size is based on surplus amount). The merit of community is successive transportation method which resources can be transported from surplus to deficient node within one physical hop. Base on ADCS architecture, the limitations in conventional system (i.e. congestion at the center, dynamic situation, etc.) can be overcome. However, to equally manipulate in resource allocation, there is a tradeoff between timeliness and operatability so dynamic setting for distribution rate which depends on the congestion is proposed. In addition, when collision occurs during community construction, the community will not expand in the collided direction but continue to expand in other directions further from original distance instead in order to substitute the collided area. So the community size will still be equivalent to original expected community size.

This paper is organized as follows: Section 2 presents application; Section 3 presents ADCS; Section 4 presents ACT, proposed technology; Section 5 shows the simulation evaluation; and Sect. 6 concludes this paper.

\section{System Application}

\subsection{Application}

Transportation system is one kind of resource utilization system applications (consists of service provision and resource allocation) which has the following characteristics:

- All resources have common objectives and functions. 


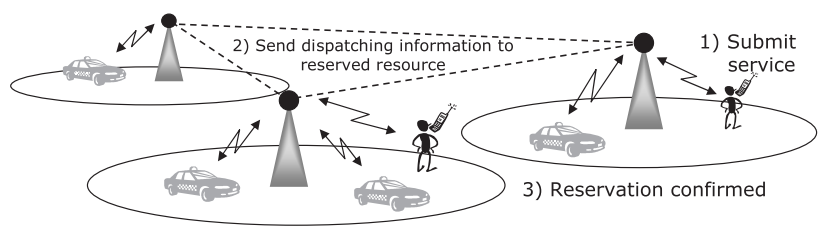

Fig. 1 Service application.

- Resources physically move in the system.

- Resource movement requires some time consumption.

Corresponding to the above characteristics, taxi dispatching system (as shown in Fig. 1) is one example of applications which has common resources. Taxi and taxi-company can be considered as resource and service provider (SP) accordingly. Each SP makes a decision on resource allocation in its area. As SP point of view, taxi should be effectively allocated without unpredictable congestion. As user point of view, service should be smoothly provided in all conditions.

\subsection{Requirements}

There are three significant issues in resource provision and utilization: service, time and quality. To overcome these issues, the system is required to meet the following requirements.

a) Online property: Online expandability, fault-tolerance, and maintenance could be assured during operation. If a node is added, fails, or stops, the others can still work properly in the system.

b) Flexibility: System needs to be able to handle with the changes in users' requests and resource mobility. Thus, flexibility is required to accommodate the changing situations.

c) Timeliness: The system is expected to allocate resource to users within a short response time.

d) Operatability: The resource allocation should be optimized because resources physically move in the system.

\section{Autonomous Decentralized Community System}

\subsection{System Architecture}

Resource provision and utilization in real implementation (when and where users will request for service) is unpredictable. Recall from Sect. 1, based on ADS concept [7], ADCS has been proposed. ADCS is a flexible system whose members autonomously coordinate to one another. In ADCS, a node autonomously constructs a group or community for service provision objective. Nodes in ADCS not only autonomously decide to join or leave a community but also independently cooperate with other members. Therefore online property and flexibility can be assured.

Figure 2 shows the structure of ADCS. Community service applications are featured with a concept of timedistance oriented system. The autonomous decision can be made by Autonomous Control Processor (ACP) that judges and processes based only on local information stored

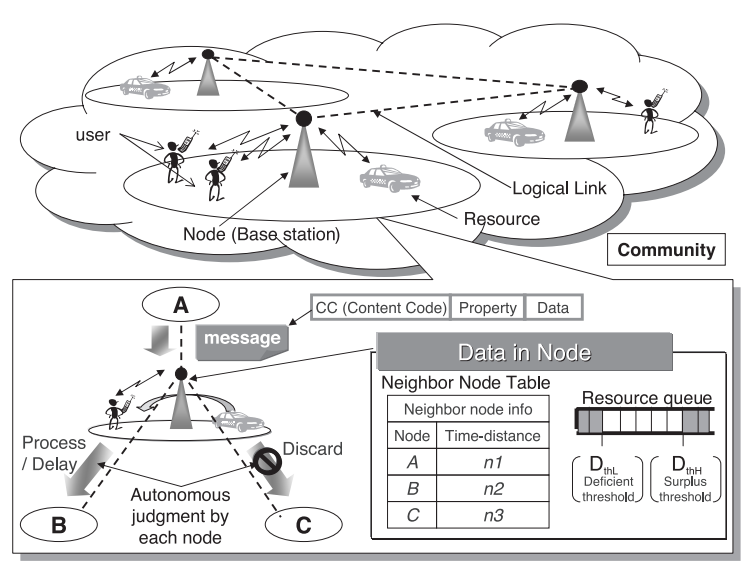

Fig. 2 Autonomous decentralized community system architecture.

in Neighbor Nodes Table (NNT). NNT indicates message directions to neighbor nodes and contains physical TimeDistance (TD) from a certain node to its neighbors. Each node represents wireless base station coverage area. It maintains wireless computers like Base Transceiver Stations (BTS), and communicates between users and resources. When each user crosses the coverage area of BTS, the mobile units hands off, or switch the communicating stations [4]. By recording the numbers of these hand offs, each station can calculate the time-distance [9], [10]. Users use mobiles to request service from the nearest node. Resource informs the nearest node when condition changes.

Each node autonomously creates and broadcasts message to neighbors. Each message-receiving node autonomously decides to process or discard the message by checking message property. Content Code (CC) contains type of message. Resource queue $\left(R_{\mathrm{N}}\right)$ indicates the number of existing resources in queue and dynamically updates in each node. $D_{\text {thH }}$ and $D_{\text {thL }}$ are thresholds indicating surplus and deficient levels which based on provision and utilization ability. A resource data in resource queue contains resource ID which uniquely identified and time stamp when the node has been notified from resource.

Based on community approach, different-objective communities are formed: 1) surplus node oriented construction for high efficiency in utilization and 2) deficient node oriented construction for high response time. Here, surplus node oriented construction is proposed.

\subsection{Successive Transportation Method}

Service provision flow simply starts when a user requests for a service. The nearest node which received the request then checks resources in queue and arranges the most appropriate resource for user by First-In-First-Out (FIFO) method. The various service levels required by users are depending on location and time.

Due to heterogeneous demands as well as resource mobility circumstance, surplus or deficiency of resources randomly appears and disappears in some nodes. In transport system, resources in each node are common and have same 


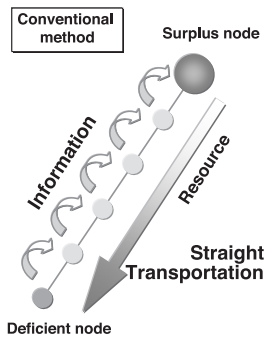

(a) Straight method

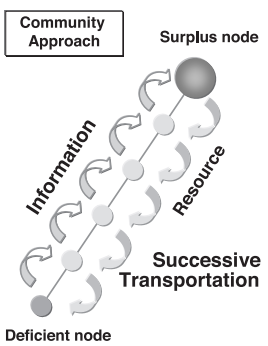

(b) Successive method
Fig. 3 Transportation method.

objectives so the resources can be moved across the nodes to serve users. Thus, to make system well-organized, resources should be distributed from surplus to deficient nodes in order to balance resource congestion in each node. However, without community and no cooperation among nodes, resources must physically move from surplus to deficient nodes. The more hops between nodes, the longer physical transportation time is consumed as shown in Fig. 3 (a).

In community, each node shares information and autonomously coordinates to one another. The transportation from surplus to deficient nodes is correspondingly understood among members in community so each node simultaneously transports resources hop by hop from surplus to deficient nodes. As a result, the transportation completes in only one physical hop. This method is called successive transportation method as shown in Fig. 3 (b).

\section{Autonomous Coordination Technology (ACT)}

Referred to the community concept which based on ADS in [14], [15] so online property and flexibility are achieved. However, the requirements are different due to different applications. The technology in [14], [15] was applied for web application which information exchanged in community. In this paper, the application is resource utilization which common physical resources are moved among nodes. Due to different applications, the technology for resource utilization is required to achieve timeliness (response time) and operatability (resource utilization efficiency) as well. Therefore, Autonomous Coordination Technology (ACT) based on community approach is proposed. ACT consists of: 1) ACT within community and 2) ACT among communities. ACT within community illustrates how a community is constructed with area limited and how resources autonomously be allocated. ACT among communities illustrates the coordination between communities when a collision occurs.

\subsection{ACT within Community}

\subsubsection{Autonomous Community Construction}

For community construction, a surplus node is a node which has vacant resources in queue more than threshold $D_{\text {thH }}$ $\left(R_{\mathrm{N}}>D_{\mathrm{thH}}\right)$; whereas, a deficient node is a node which has resources in queue less than threshold $D_{\mathrm{thL}}\left(R_{\mathrm{N}}<D_{\mathrm{thL}}\right)$.
A node beside these two conditions $\left(D_{\mathrm{thL}} \leq R_{\mathrm{N}} \leq D_{\mathrm{thH}}\right)$ is called a normal node. To balance resource congestion, a surplus node autonomously constructs community by calculating community size and broadcasting community construction message to neighbors. Each node which received construction message autonomously decides to process or discard the message based on the content in construction message and the time-distance information in NNT. As community membership, a member node which is in deficient state can request resources from the community surplus node.

a) Community area determination: Again, the goals of this paper are achieving timeliness and operatbility assurance. In resource utilization, resource distribution time depends on two factors: how many surplus resources in surplus node, and how long physical resource movement takes. Thus surplus level is used as one parameter to determine community size. Moreover, the size should be limited because of two reasons: 1) the community cannot expand endlessly otherwise a request probably cannot be served due to long distance; 2) each node can belong to only one community or it may receive duplicate resources from different communities. As of these concerns, community size is limited. On the other hand, resource movement time is obtained by timedistance instead of numbers of hops because the distance between each hop-pair is naturally different. To fairly allocate resources for each boundary node in all directions, time-distance is used as another parameter, and obtained by using the average time-distance from surplus node to its neighbor nodes. Hence, a node which changes to surplus state autonomously calculates CommSize by the following formula:

$$
\text { CommSize }=\frac{\sum_{i=1}^{N_{\mathrm{N}}} T D(i)}{N_{\mathrm{N}}} \times\left(R_{\mathrm{N}}-D_{\mathrm{thH}}\right),
$$

where CommS ize is community size, $T D(i)$ is time-distance between surplus node and its neighbor node $i, N_{\mathrm{N}}$ is the number of surplus's neighbor nodes, and $R_{\mathrm{N}}$ is the amount of resources in surplus node.

b) Community construction message: Once surplus node determines the community size, it will transmit community construction message to neighbors in the following format:

\begin{tabular}{|l|l|l|l|l|l|}
\hline CC & ID & SurplusID & Sender & CommSize & $C T D$ \\
\hline
\end{tabular}

Each node which receives construction message autonomously adds up time-distance from surplus node and updates into cumulative time-distance field, $C T D$, and then compares to CommSize. If the CTD $\leq$ CommSize, the certain node will decide to join community and forward updating message to lower-level adjacent nodes; otherwise it will discard the message. As a result, a logical connection among group of equivalent time-distance nodes is formed and called as community network.

Figure 4 shows community construction technique. 'A' has surplus in resources so it calculates CommSize and transmits construction message to neighbors. Each node which receives the message autonomously makes a decision 


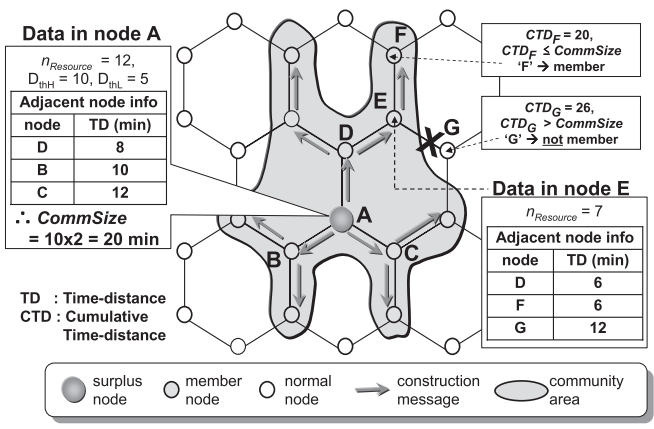

Fig. 4 Autonomous community construction.

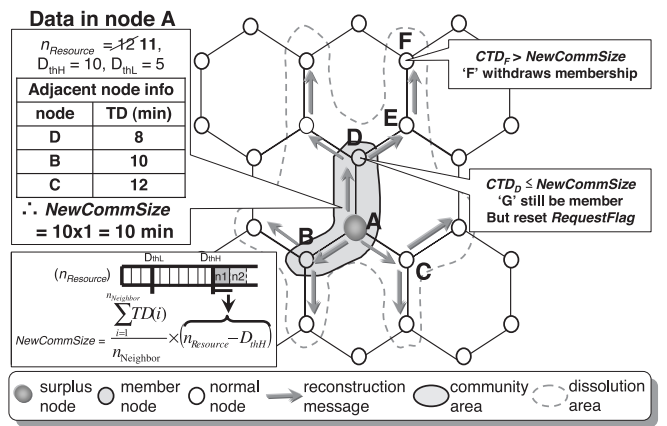

Fig. 5 Autonomous community reconstruction and dissolution. to process or discard it. For example, 'D', 'E', and 'F' have $C T D \leq C o m m S i z e$, so they decide to join the community and forward the message to lower-level nodes. In contrast, ' $G$ ' has $C T D>C o m m S$ ize so it decides to discard the message. As a result, the community area is limited.

c) Community management: A member node which is deficient in resources can request resources from surplus node. But if the surplus node immediately dispatches resources once receiving a request, the quantity of surplus resources as well as community size will naturally decrease. As a result, previous member nodes at community boundary may suddenly be excluded from community and cannot receive any resources even if requested. To avoid this situation, surplus node sets up a request acceptance period called session. Once surplus node sends construction message, it will wait for a session to collect requests and later on decide for an allocation. As time-distance affects message delivery time, the session should be increased by the community size. Thus a session is obtained by a constant value multiplied by the amount of surplus resources. In each session, to avoid single monopoly, a deficient node will send a request only once and set a flag called RequestFlag to indicate a request waiting state. RequestFlag will be reset by each node when receiving the next reconstruction message. Autonomous management process improves not only resource provision, but also resource utilization efficiency.

d) Community reconstruction and dissolusion: In each session, $R_{\mathrm{N}}$ changes due to resource allocation or the dynamic change itself, then surplus node re-calculates the CommSize. If new CommSize (CommSize') not equal to zero, surplus node will send reconstruction message and reform community expansion similar to construction process. However if CommSize' decreases to zero, surplus node will send dissolution message instead. Each node which receives reconstruction message autonomously forwards to lower-level nodes and compares the CommS ize to its $C T D$. If CommSize $e^{\prime}>C T D$, it will keep the membership. In contrast, if CommSize' $\leq C T D$, it will withdraw from membership and become a normal node. Finally, each node which receives dissolution message then changes to normal node. In Fig. 5, 'A' re-calculates for CommS ize' and transmits reconstruction message. 'D' has $C T D \leq$ CommSize' so it remains a member; whereas ' $\mathrm{E}$ ' and ' $\mathrm{F}$ ' have $C T D>$ CommS ize' so they leave the community.

\subsubsection{Autonomous Resource Allocation}

a) Request rate: Among members in one surplus community, more than one node may be deficient in resources and simultaneously request resources to surplus node. How many resources each deficient node should receive to be able to serve user as soon as possible (timeliness) and surplus node should allocate to fairly distribute resources to each deficient node (operatability)? To address this challenge, the variation in resource distribution rate is studied. The actual amount of resources distributed from surplus node to each deficient node is given by:

$$
\text { ActualResource }=\left(R_{\mathrm{N}}-D_{\text {thH }}\right) \times \text { ReqRate, }
$$

where ReqRate is a constant rate that surplus node sets to weigh resource distribution to deficient node. To derive ReqRate, Eq. (2) is re-written in terms of the percentage of deficiency as follows:

$$
\text { ReqRate }=\frac{R_{\mathrm{Q}}}{R^{-}}
$$

where $R_{\mathrm{Q}}$ is the number of requested resources from surplus node, and $R^{-}$is the amount of total deficient resources in a deficient node.

To obtain the appropriate ReqRate under changing environment, ReqRate should not be a fixed value as of the following reasons:

- High request rate: ReqRate is set to 1 (set to the total deficiency). If a surplus node provides many resources to the first coming requesters, resources will be distributed quickly but the allocation is unfair to some deficient nodes which came in late due to long distance. In other words, the operatability is low even though timeliness is high in case of high ReqRate.

- Low request rate: ReqRate is set to one resource. If a surplus node provides few resources at a time, the allocation to each deficient node is fair but it may have to distribute resources several times. So the operatability is high but timeliness is low in case of low ReqRate. 


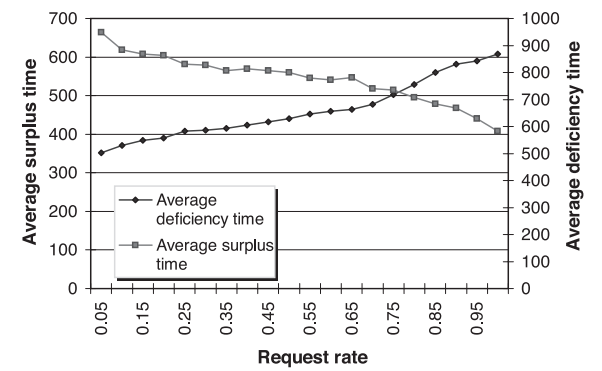

Fig. 6 Tradeoff between timeliness and operatability.

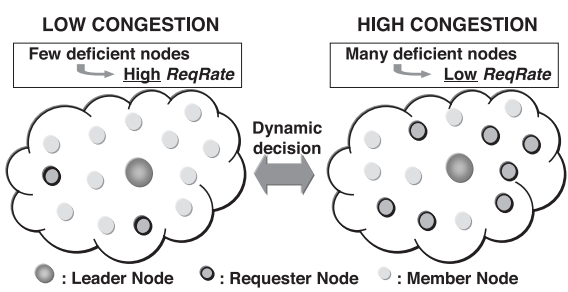

Fig. 7 Autonomous community control.

b) Tradeoff between timeliness and operatability: The relationship between timeliness and operatability versus ReqRate is simulated. Timeliness is measured by the average deficiency time; whereas operatability is measured by the average surplus time in system.

Timeliness $(\mathrm{Ti})$ and Operatability $(\mathrm{OP})$ are given by:

$$
T i=\frac{\sum_{i=1}^{N_{\mathrm{S}}} t^{-}(i)}{N_{\mathrm{S}}} \quad \text { and } \quad O p=\frac{\sum_{i=1}^{N_{\mathrm{S}}} t^{+}(i)}{N_{\mathrm{S}}},
$$

where $t^{-}(i)$ is time usage to fulfill deficiency of a deficient node $i, t^{+}(i)$ is time usage to dissolve resource surplus of a surplus node $i$, and $N_{\mathrm{S}}$ is a number of nodes in the system.

The computational simulation result using the proposed method is shown in Fig. 6. In this simulation, the system configures of 20 hops of network radius, $5000 \mathrm{log}$ ical seconds of total simulation time. Each node holds 50 resources. Users arrival rate is 30 users/minute. The result could affirm a tradeoff between timeliness and operatability and is also enough to find the dynamic setting for ReqRate. c) Autonomous community control: Again, toward the goals, resources should be promptly and evenly utilized. In each community, surplus and deficiency level differs due to unpredictable environments. To accomplish well-organized, a dynamic set for ReqRate depending on the congestion level at the moment is proposed. In decentralized system, each individual node does not know the entire situation in the system; however, a surplus node can understand request congestion in its own community based on local information from request messages. Regarding this approach, surplus node sets high ReqRate in low request congestion and vice versa to balance surplus and deficiency level in community. Figure 7 shows the idea of the approach. Surplus node dynamically sets ReqRate according to the current situation. In the left community, only few deficient nodes request for resources so surplus node autonomously sets high ReqRate to each requester. In contrast, many deficient nodes request for resources in the right community so surplus node sets low ReqRate to each requester. Base on community control in ACT, timeliness and operatability can be assured.

d) Request aggregation: When a member becomes deficient in resources, it autonomously informs to upper-level node and so on until reach community surplus node. Surplus node will wait for a session to aggregate requests from all members before making each resource allocation.

e) Community request rate calculation: At this point, a surplus node knows the request congestion level according to request aggregation. Thus, surplus node sets request rate for its own community by evenly dividing the surplus resources by the total deficient resources as follows:

$$
\text { CommReqRate }=\frac{\left(R_{\mathrm{N}}-D_{\mathrm{thH}}\right)}{\sum_{i=1}^{N^{-}} R_{\mathrm{Q}}(i)},
$$

where CommReqRate is community request rate, $R_{\mathrm{Q}}(i)$ is the number of deficient resources in a deficient node $i$, and $N^{-}$is the amount of deficient nodes in community.

Note that if CommReqRate > 1, it will be set to 1

f) Resource distribution: Surplus node autonomously determines resource distribution for each deficient node based on the above calculated CommReqRate, which can be considered as the community dynamic weight for resource allocation, as the following formula:

$$
R_{\mathrm{D}}(i)=\text { CommReqRate } \times R_{\mathrm{Q}}(i),
$$

where $R_{\mathrm{D}}(i)$ is the number of resources distributed from surplus node to deficient node $i$.

Surplus node shares this information to all members. Each node understands the same situation so a node which has to deliver resources autonomously starts transporting resources and informs the others at the same time. Thus the resources are successively transported and simultaneously moved from surplus to deficient nodes in only one physical hop as described in Sect. 3.2.

\subsection{ACT among Communities}

During community construction, there might be a nearby community already exists and causes a collision. Referred to Sect. 4.1.1.a), each node can belong to only one community to avoid duplicate resource provision. So the community construction toward collision direction is prohibited. In this case, the community should expand more to other directions to compensate the shortage area and make the area size be equivalent as shown in Fig. 8.

a) Community collision detection: When a member of one community receives another construction message, it will check surplus node ID contained in the message. If the message is generated by different surplus node, an existing member autonomously detects a collision (Fig.9(a)), then compares CommSize and CTD. If CTD>CommSize, it will discard the message. But if $C T D \leq C o m m S i z e$, it autonomously generates a collision detection message reply to a sender (Fig. 9 (b)) in the following format: 


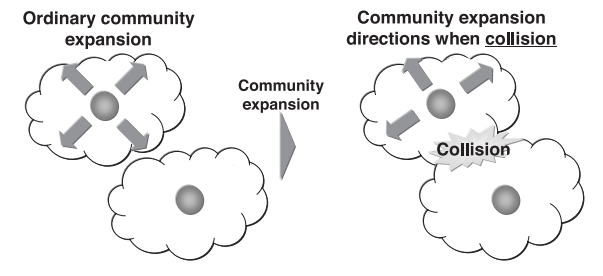

Fig. 8 Autonomous coordination.

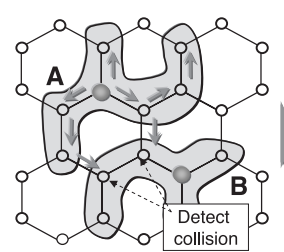

(a)

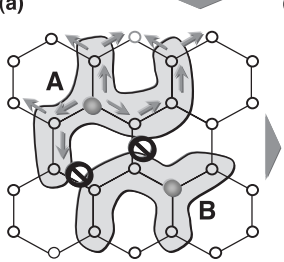

(c)
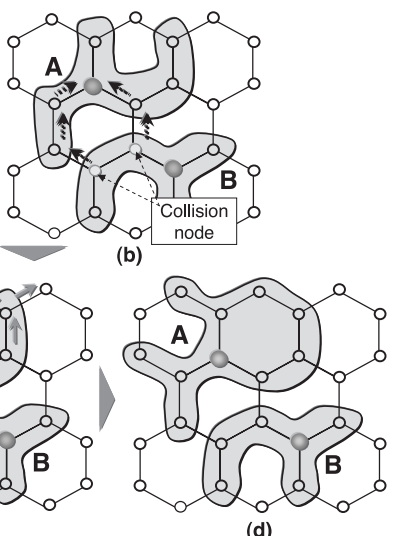

(d)

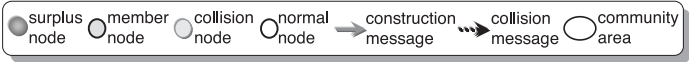

Fig. 9 Autonomous coordination step.

\begin{tabular}{|l|l|l|l|l|l|}
\hline CC & ID & CollisionID & SurplusID & Sender & $R T D$ \\
\hline
\end{tabular}

where RTD is remaining time-distance which is obtained from CommSize minus by $C T D$.

b) Collision community expansion: During community construction, if a surplus node receives a collision detection message, it will re-calculate for CommSize' based on RTD retrieved from the message and retransmits construction message with CommSize $e^{\prime}$ in the following format (Fig. 9 (c)):

\begin{tabular}{|l|l|l|l|l|l|}
\hline CC & ID & SurplusID & Sender & CommSize $^{\prime}$ & CTD \\
\hline
\end{tabular}

Where CommSize' is a new CommS ize re-calculated by using the product of original CommS ize and $R T D$ as follows:

$$
\text { CommSize } e^{\prime}=\frac{\sum_{i=1}^{N_{\mathrm{N}}} T D(i)}{N_{\mathrm{N}}} \times\left(R_{\mathrm{N}}-D_{\mathrm{thH}}\right)+R T D .
$$

c) Community reconstruction: Each member receives new construction message generated by its community surplus node, it will compare CommSize' and CTD then autonomously decide to expand the community by forwarding the message to lower-level nodes. As a result, a community finally constructs with equivalent size (Fig. 9(d)).

\section{Evaluation}

\subsection{Timeliness and Operatability}

The objective of evaluation is to prove that the proposed
Table 1 Simulation parameters.

\begin{tabular}{lll}
\hline Parameter & Description & Value [Unit] \\
\hline SimTime & Total simulation time & $50000[\mathrm{~s}]$ \\
Radius & Network radius & $20 \mathrm{hops}$ \\
CongestionRate & Congestion rate & $0 \sim 0.1[\%]$ \\
TimeDist & Time-distance between nodes & $180[\mathrm{~s}]$ \\
$D_{\mathrm{thH}}$ & Surplus threshold & 65 \\
$D_{\mathrm{thM}}$ & Average resources maintain in node & 50 \\
$D_{\mathrm{thL}}$ & Deficient threshold & 35 \\
ShufflePeriod & Congestion levels change period & $0 \sim 3000[\mathrm{~s}]$ \\
SessionTime & Acceptance period & $20[\mathrm{~s}]$ \\
SuppressionRate & Suppression rate & $20[\%]$ \\
\hline
\end{tabular}

technology is effective in terms of timeliness and operatability in the application which has common resources, i.e. taxi dispatching system. So a simulation based on the taxi system in Tokyo is made and the evaluation is based on simulation assumption. Timeliness and operatability given in (4) are used as indicators. The simulation uses a hexagonal mesh network as a network topology because many of the actual networks for base stations of wireless communication forming networks in the similar way. Table 1 shows main simulation parameters which is corresponding to the assumption time and logical time for simulation.

For evaluating Timeliness and Operatability, a comparison of: 1) ADCS with ACT (proposed technology), 2) ADCS without ACT and each nodes request rate set to 1.0, and 3) Centralized system (conventional technology), is made. The result is the average value of 50 times simulations. Figure 10 (a) shows deficient time which represents timeliness evaluation. Figure 10 (b) shows surplus time which represents operatability evaluation. The $X$-axis is the number of users per minute. The Y-axis is the number of average congestion time. The result shows that the congestion time is low at low user rate, and highly increase at high user rate in centralized system because the centralized center's ability is limited, and causes the congestion. On the other hand, congestion time in ADCS follows a near linear increase at all rates. As a result, the effectiveness in ADCS is improved at high user rate ( $>59$ users/min in timeliness evaluation and $>45$ users/min in operatability evaluation). In addition, ADCS with ACT shows lower congestion time comparing to ADCS without $\mathrm{ACT}$ at any user rate.

\subsection{Standard Deviation}

Due to unpredictable congestion, each node expects to maintain balanced resource to median level as much as possible. Here, the standard deviation of the system is also evaluated to show the stability of the system. $S D$ is given by:

$$
S D=\sqrt{\frac{\sum_{i=1}^{N_{\mathrm{S}}}\left(R_{\mathrm{N}}(i)-\frac{\sum_{i=1}^{N_{\mathrm{S}}} R_{\mathrm{N}}(i)}{N_{\mathrm{S}}}\right)^{2}}{N_{\mathrm{S}}}} .
$$

The $S D$ of resources in base station versus user rate is evaluated with the following simulation conditions: user rate varies from $0-90$ users/min, request rate is 1.0 , and simulation time is 100 times. 


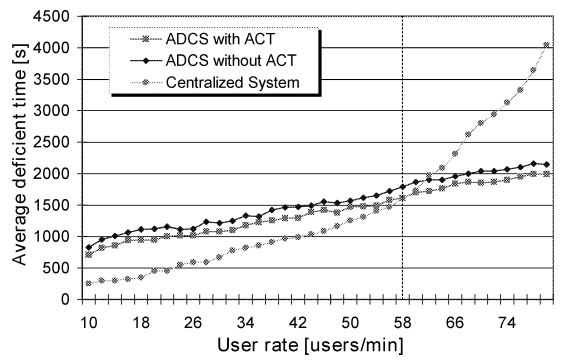

(a) Timeliness evaluation

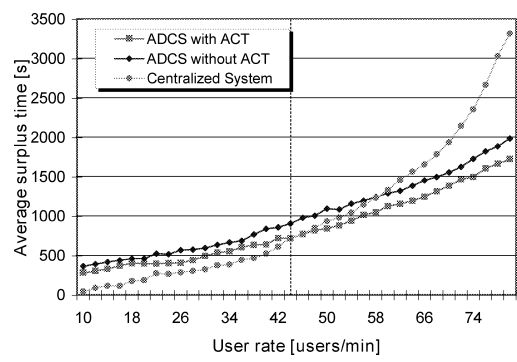

(b) Operatability evaluation

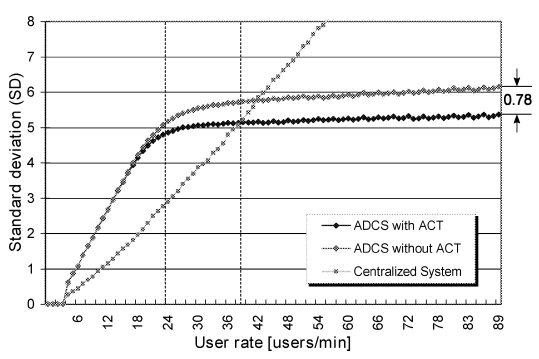

(c) SD evaluation of ACT

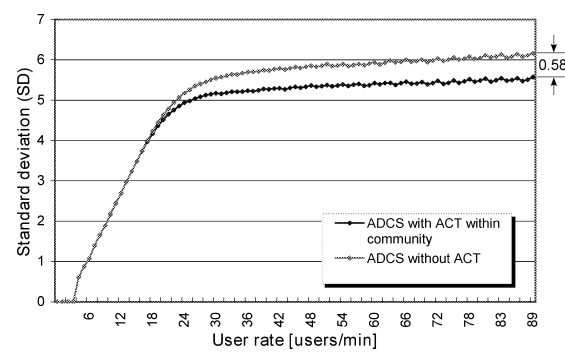

(d) SD evaluation of ACT within community

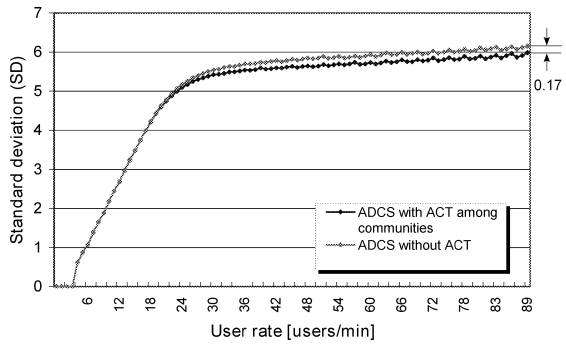

(e) SD evaluation of ACT among communities

Fig. 10 Evaluation results.

Figure 10 (c) shows a comparison of: 1) ADCS with ACT, 2) ADCS without ACT, and 3) centralized system. Figure 10 (c) presents that $S D$ of centralized system follows a linear increase at any user rate; in ADCS without ACT, $S D$ follows a linear increase till around 24 users/min and then remains steady; and in ADCS with ACT, the result is similar to ADCS without ACT but $S D$ is lower in steady state. At 90 users/min, ADCS with ACT shows $0.78 S D$ lower than ADCS without ACT. Hence, the stability is improved by proposed technology at high user rate ( $>38$ users $/ \mathrm{min}$ ). Figure 10 (d) presents the stability improvement by ADCS with ACT within community and the result shows that ADCS with ACT within community has $0.58 S D$ lower than ADCS without ACT at 90 users/min. Figure 10 (e) presents the stability improvement by ADCS with ACT among communities and the result shows that ADCS with ACT among community has $0.17 S D$ lower than ADCS without ACT at 90 users/min.

\section{Conclusion}

The resource utilization under dynamic changing environment requires a system which satisfies online property, flexibility, timeliness and operatability. Based on community approach, online property and flexibility can be satisfied but it is difficult to assure timeliness and operatability at the same time. Thus, this paper proposes Autonomous Coordination Technology (ACT) through community organization which based on Autonomous Decentralized Community System (ADCS). ACT consists of the coordination within community and among communities. ACT within community presents autonomous community construction, and resource allocation according to a tradeoff between timeliness and operatability. Because of community, the resources can be transported by successive transportation method in one physical hop. On the other hand, ACT among communities presents the coordination when community collision occurs. Finally, the simulation is made to evaluate the proposed technology in terms of timeliness and operatability assurance. Timeliness and operatability results show the effectiveness of proposed technology is improved at high user rate ( $>59$ users/min in timeliness and $>45$ users/min in operatability). The stability of the system is evaluated by $S D$ in a node. $S D$ results show a linear increasing in conventional system at any user rate but a steady state in proposed technology at high user rate (> 24 users/min). In conclusion, the proposed technology improves timeliness and operatability in a large-scale resource utilization system.

\section{Acknowledgements}

This work is partly supported by National Institute of Information and Communications Technology, Japan under Early-concept Grants for Exploratory Research on Newgeneration Network (No.145-9).

\section{References}

[1] M. Weiser, The computer for the 21st century, Scientific American, 1991.

[2] M. Weiser, "Some computer science issues in ubiquitous computing," Commun. ACM, vol.36, no.7, pp.75-84, July 1993.

[3] "Ministry of land, infrastructure, transport and tourism." http://www.mlit.go.jp/road/ITS/topindex/topindex_g02_1.html

[4] C.C. Tao and C.Y. Chen, "Heuristic algorithms for the dynamic taxipooling problem based on intelligent transportation system technologies," FSKD '07: Proc. Fourth International Conference on Fuzzy Systems and Knowledge Discovery, pp.590-595, IEEE Computer Society, Washington, DC, USA, 2007.

[5] M. Takagi and I. Masaki, "Priority-controlled networks in ITS," SICE 2003 Annual Conference, pp.2390-2393, Aug. 2003. 
[6] D.W. Lee, E.J. Bass, and S.D. Patek, "Towards a transportation network model for air taxi dispatch planning," 2005 IEEE Systems and Information Engineering Design Symposium, pp.293-301, April 2005.

[7] K. Mori, "Autonomous decentralized systems: Concept, data field architecture and future trends," Proc. International Symposium on Autonomous Decentralized Systems (ISADS '93), pp.28-34, IEEE Comput. Soc. Press, 1993.

[8] G. Sun and K. Mori, "Autonomous fading and navigation for information allocation and search under evolving service system," Asia Pacific Symposium on Information and Telecommunication Technologies (APSITT '99), pp.326-330, Aug. 1999.

[9] T. Ono, K. Ragab, N. Kaji, and K. Mori, "Service oriented communication technology for achieving assurance," ICDCSW '02: Proc. 22nd International Conference on Distributed Computing Systems, pp.69-74, IEEE Computer Society, Washington, DC, USA, 2002.

[10] N. Kaji, K. Ragab, T. Ono, and K. Mori, "Autonomous synchronization technology for achieving real time property in service oriented community system," 2nd International Workshop on Autonomous Decentralized System, pp.16-21, IEEE Comput. Soc., 2002.

[11] K. Hama, Y. Horikoshi, H. Endo, B.P. Kloester, and X. Lu, "Autonomous community integration and division technology for high response service," International Symposium on Autonomous Decentralized Systems, vol.0, pp.437-444, 2007.

[12] K. Mahmood, X. Lu, and K. Mori, "Autonomous community construction technology to achieve service assurance in ADCS," IEICE Trans. Inf. \& Syst., vol.E91-D, no.9, pp.2259-2266, Sept. 2008.

[13] F. Wei, K. Hama, Y. Kotake, X. Lu, and K. Mori, "Autonomous collaboration technology in resource provision and utilization community," 2009 International Symposium on Autonomous Decentralized Systems, pp.1-7, IEEE, March 2009.

[14] Y. Horikoshi, K. Ragab, N. Kaji, and K. Mori, "Service discovery technology in autonomous decentralized community system," AsiaPacific Symposium on Information and Telecommunication Technologies (APSITT 2003), pp.563-568, Nov. 2003.

[15] Y. Sugiyama, Y. Horikoshi, H. Kuriyama, and K. Moriyama, "Service search technology in autonomous decentralized community system for timeliness," Proc. International Symposium on Autonomous Decentralized Systems (ISADS'05), pp.336-343, IEEE, 2005.

[16] A. Shimura, T. Sakaibara, M. Hiraiwa, and T. Aizono, "Proposal of an autonomous group-management model and its application to intelligent transport system," Proc. Sixth International Symposium on Autonomous Decentralized Systems (ISADS'03), pp.71-79, 2003.

[17] K. Ragab, N. Kaji, and K. Mori, "Acis: A large-scale autonomous decentralized community communication infrastructure," IEICE Trans. Inf. \& Syst., vol.E87-D, no.4, pp.936-946, April 2004.

[18] K. Ragab and K. Mori, "Acis-hierarchy: Enhancing community communication delay for large-scale information systems," IEICE Trans. Commun., vol.E87-B, no.7, pp.1797-1805, July 2004.

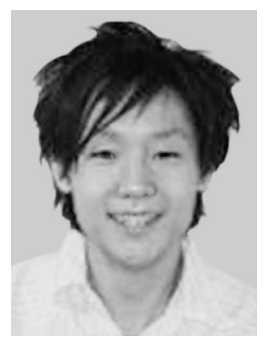

Kotaro Hama had joined and received M.S. degree in Computer Science from Department of Computer Science, Tokyo Institute of Technology in 2005 and 2007, respectively.

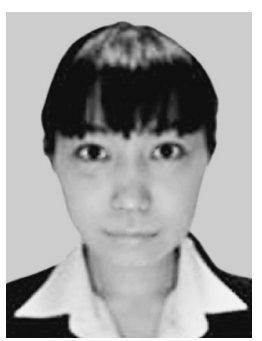

Riyako Sakamoto has joined Department of Computer Science, Tokyo Institute of Technology as master student since 2008. Her research interests include autonomous decentralized community systems. She is a student member of IEEE.

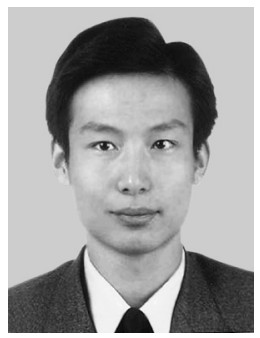

Xiaodong Lu received the BS degree in electronic engineering in 1997, the MS degree in computer science in 2000, both from Lanzhou University, P.R.China, and $\mathrm{PhD}$ degree in computer science from Tokyo Institute of Technology, in 2005. He is an assistant professor in the Department of Computer Science at Tokyo Institute of Technology. His research interests include distributed and high-assurance information systems and mobile agent. He is a member of IEEE and IEEE CS.

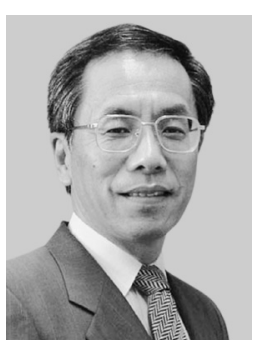

Kinji Mori received the B.S., M.S. and $\mathrm{Ph} . \mathrm{D}$. degrees in the Electrical Engineering from Waseda University, Japan in 1969, 1971 and 1974, respectively. From 1974 to 1997 he was in System Development Lab., Hitachi, Ltd. In 1997 he joined Tokyo Institute of Technology, Tokyo, Japan as a professor. His research interests include the distributed computing, the fault tolerant computing and the mobile agent. $\mathrm{He}$ is a Fellow of IEEE and a member of IPSJ and SICE, Japan.

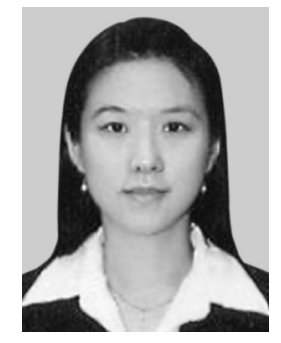

Titichaya Thanamitsomboon received the B.S. and M.S. degrees in Electrical Engineering from Chulalongkorn University, Bangkok, Thailand in 1999 and 2002, respectively. She joined Department of Computer Science, Tokyo Institute of Technology as Ph.D. candidate under supervision of Prof. Kinji Mori in 2009. Her research interests include autonomous decentralized community systems, and distributed applications. 